\title{
Understanding, explaining, and utilizing medical artificial intelligence
}

\author{
Romain Cadario $\oplus^{1 凶}$, Chiara Longoni $\oplus^{2}$ and Carey K. Morewedge $\circledast^{2}$
}

\begin{abstract}
Medical artificial intelligence is cost-effective and scalable and often outperforms human providers, yet people are reluctant to use it. We show that resistance to the utilization of medical artificial intelligence is driven by both the subjective difficulty of understanding algorithms (the perception that they are a 'black box') and by an illusory subjective understanding of human medical decision-making. In five pre-registered experiments (1-3B: $N=2,699)$, we find that people exhibit an illusory understanding of human medical decision-making (study 1). This leads people to believe they better understand decisions made by human than algorithmic healthcare providers (studies 2A,B), which makes them more reluctant to utilize algorithmic than human providers (studies 3A,B). Fortunately, brief interventions that increase subjective understanding of algorithmic decision processes increase willingness to utilize algorithmic healthcare providers (studies 3A,B). A sixth study on Google Ads for an algorithmic skin cancer detection app finds that the effectiveness of such interventions generalizes to field settings (study 4 : $N=14,013$ ).
\end{abstract}

A lgorithms are rapidly diffusing through healthcare systems ${ }^{1}$, providing support for outpatient services (for example, telehealth) and supply to match demand for inpatient care services $^{2-4}$. Algorithmic-based healthcare services (medical 'artificial intelligence' (AI)) are cost-effective and scalable and provide expert-level accuracy in applications ranging from the detection of skin cancer ${ }^{5}$ and emergency department triage ${ }^{6,7}$ to diagnoses of COVID-19 from chest X-rays ${ }^{8}$. Adoption of medical AI is critical for providing affordable, high-quality healthcare in both the developed and developing world ${ }^{9}$. However, large-scale adoption of AI hinges not only on adoption by healthcare systems and providers but also on patient utilization, and patients are reluctant to utilize medical $\mathrm{AI}^{10-12}$. Patients view medical $\mathrm{AI}$ as unable to meet their unique needs ${ }^{10}$ and as performing more poorly than comparable human providers $^{12}$ and feel it is harder to hold AI providers accountable for mistakes than comparable human providers ${ }^{11}$.

We propose that another important barrier to adoption of medical AI is its perceived opacity, that it is a 'black box ${ }^{13,14}$. People do not subjectively understand how algorithms make medical decisions, and this impairs their utilization. Moreover, we suggest that this barrier does not rest solely on the perceived opacity of AI. It is also grounded in an illusory understanding of the medical decisions made by human providers. We theorize that people erroneously believe they better understand the decision-making of human than AI healthcare providers, and this erroneous belief contributes to algorithm aversion in healthcare utilization (greater reluctance to utilize healthcare delivered by AI providers than by comparable human providers).

Our theory is grounded in a reality (objective knowledge: what people actually know) and an illusion (subjective knowledge: what people believe they know). Decision processes used by algorithms are perceived to be opaque by patients, because they are often actually opaque to medical professionals and even their creators, who cannot explain them ${ }^{15,16}$. In other words, subjective and objective knowledge of algorithmic decision processes are similarly poor. We argue that decisions made by human providers appear more transparent, but this perception is an illusion; subjective knowledge of human decision processes is greater than objective knowledge. The perceived transparency of human decision-making stems from a belief that introspection provides direct access into the psychological processes by which people make decisions ${ }^{17}$. But people actually lack access to their own associative machinery ${ }^{18,19}$, and rely on heuristics to understand the decision-making of other people ${ }^{20}$. Human decision-making is often as much a black box as decisions made by algorithms.

We suggest that these coupled effects make people aware of their limited understanding of medical decisions made by algorithmic providers, but also lead them to overestimate their understanding of (similarly opaque) decisions made by human providers. Indeed, we find that people claim greater subjective understanding for medical decisions made by human than AI providers (people feel that they know more), but they exhibit similar objective understanding of decisions made by both providers (people actually know as little about both decision processes). We propose that, in turn, poorer subjective understanding of decisions made by AI than human healthcare providers underlies reluctance to utilize medical AI. Subjective understanding plays a critical role in the adoption of innovations, from new policies to consumer products, and facilitates adoption even when people have little objective understanding of their benefits or how innovations work ${ }^{21,22}$.

We tested our predictions in a set of five pre-registered online experiments with nationally representative and convenience samples (studies 1, 2A,B and 3A,B: $N=2,699$ ), and a sixth pre-registered online field study on Google Ads (study 4: $N=14,013$ ). We find that subjective understanding drives healthcare provider utilization. Moreover, we find that greater subjective understanding of medical decisions made by human than AI healthcare providers contributes to algorithm aversion, but it can be remediated with cost-effective interventions that increase utilization of algorithmic providers without reducing the utilization of human providers. All experiments were approved for use with human subjects by institutional review boards. All conditions, measures and exclusions are reported; data and pre-registrations are available at https://osf.io/taqp8/.

'Rotterdam School of Management, Erasmus University, Rotterdam, the Netherlands. ${ }^{2}$ Questrom School of Business, Boston University, Boston, MA, USA. 凶e-mail: cadario@rsm.nl 


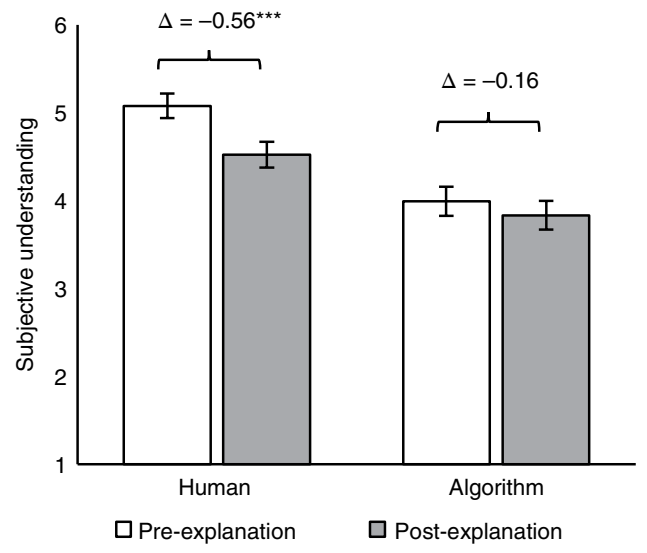

Fig. 1 | Illusory understanding of human decision-making in study $\mathbf{1}$. Making people explain provider decision processes reduces their subjective understanding of decisions made by human providers, but not decisions made by algorithmic providers (study $1 ; N=297$ ). Error bars \pm 1 s.e.m.

\section{Results}

Study 1. Our theory proposes that the greater subjective understanding people claim for medical decisions made by human than algorithmic healthcare providers is due to illusory understanding of decisions made by human providers. Making people explain a medical decision made by a human or algorithmic provider should then reduce subjective understanding of that decision more when the provider is human.

We recruited a nationally representative online sample of 297 US residents (mean age 45.37 years, $53 \%$ female) from Lucid. We used a 2 (provider: human, algorithm) $\times 2$ (rating order: pre-intervention, post-intervention) mixed design. Participants were randomly assigned to one of the two provider conditions between subjects, and ratings of subjective understanding were repeated within subjects.

We first described triage of a potentially cancerous skin lesion with visual inspection and referred to the healthcare provider as a "doctor" or "algorithm" depending on provider condition, for example, "A doctor [algorithm] will examine the scans of your skin to identify cancerous skin lesions." If risk was assessed to be high, the provider would refer the patient to a dermatologist to determine the appropriate course of action (see Supplementary Appendix 1 for introductory stimuli used in all studies).

Next, all participants provided a first rating of their subjective understanding of the provider's decision-making with a measure adapted from relevant prior research ${ }^{22,23}$ ("To what extent do you understand how a doctor [algorithm] examines the scans of your skin to identify cancerous skin lesions?" with 1 for not at all and 7 for completely). To test the degree to which this rating was illusory, participants then generated a mechanistic explanation of the provider's decision process (see Supplementary Appendix 2 for instructions). Similar interventions have been successfully implemented to shatter the illusion of understanding in other domains ${ }^{23}$. After completing the intervention, participants provided a second rating of their subjective understanding of provider's decision process. Greater reductions in pre-intervention to post-intervention ratings indicate a larger illusion of understanding.

To test whether participants exhibited a greater illusion of explanatory depth for the decision processes used by human than algorithmic providers, we regressed subjective understanding on provider ( 0 for algorithm, 1 for doctor), rating order ( 0 for pre-intervention, 1 for post-intervention) and their interaction. The analyses revealed the predicted significant interaction $\left(\Delta_{\mathrm{H}}-\Delta_{\mathrm{A}}=-0.40, z=-2.61, P=0.009\right.$; Fig. 1$)$. Participants claimed greater subjective understanding of a human than an algorithmic decision processes (pre-intervention: $\beta=1.08, z=5.02, P<0.001$; post-intervention: $\beta=0.69, z=3.19, P=0.001$ ). More importantly, the intervention significantly reduced reported subjective understanding of decisions made by human providers $\left(M_{\mathrm{pre}}=5.08\right.$ versus $\left.M_{\text {post }}=4.52, \Delta_{\mathrm{H}}=-0.56, z=-5.27, P<0.001\right)$, but not decisions made by algorithmic providers $\left(M_{\text {pre }}=3.99\right.$ versus $M_{\text {post }}=3.83$, $\left.\Delta_{\mathrm{A}}=-0.16, z=-1.47, P=0.14\right)$. These results hold when including all control variables in the regression (Supplementary Appendix 3).

Studies 2A,B. In studies 2A,B, we directly test our theory against an obvious alternative explanation, that greater subjective understanding for human than algorithmic healthcare providers is due to differences in objective understanding. We predicted that people claim greater subjective understanding of decisions made by human than algorithmic providers, but possess a similar objective understanding of decisions made by human and algorithmic providers.

We recruited nationally representative samples of US residents (2A: $N=400, M_{\text {age }}=45.36,52 \%$ female; $2 \mathrm{~B}: N=403, M_{\text {age }}=45.16$, $52 \%$ female) from Lucid. Participants were randomly assigned to one of four conditions in a 2 (provider: human, algorithm) $\times 2$ (understanding type: subjective, objective) between-subject design.

In the same skin cancer detection context as study 1, we manipulated whether the provider was a "primary care physician" or "machine learning algorithm". Next, we measured either objective or subjective understanding of that medical decision.

In objective understanding assessment conditions, we measured participants' actual understanding of the process by which providers identity cancerous skin lesions. To do this, we developed a quiz in consultation with experts in the relevant medical domain (preliminary visual inspection of moles): a team of dermatologists at a medical school in the Netherlands, and a team of developers of a popular skin cancer detection application in Europe. We identified at least three objective differences in the approach used by primary care physicians versus machine learning algorithms to perform a visual inspection. First, primary care physicians generally consider a predetermined set of features such as the lesion's asymmetry, border, colour and diameter ${ }^{24}$, whereas machine learning algorithms do not need to extract these visual features. They use raw pixels to determine visual similarity between the lesion and other lesions ${ }^{5}$. Second, primary care physicians generally use a decision tree in which mole features are considered separately and often sequentially ${ }^{25,26}$, whereas machine learning algorithms consider all features simultaneously. Third, primary care physicians make a binary decision, assigning the combination of visual symptoms to a low-risk or a high-risk category, whereas machine learning algorithms compute a more granular probability of malignancy ${ }^{5}$, although medical AI services may communicate a dichotomized low/high risk result to patients.

Building on these three differences, we created a three-item multiple-choice test intended to assess objective understanding. Each test item had one correct answer for human providers, one (different) correct answer for machine learning algorithms and one incorrect distractor answer (Cronbach's $\alpha=0.65$ in the human condition and $\alpha=0.67$ in the algorithm condition). We scored objective understanding by summing the correct answers; thus, scores ranged from 0 to 3 (algorithm condition: $m=1.33$, s.e. 0.09 , human condition: $m=1.34$ and s.e. 0.09 ).

In subjective understanding assessment conditions in study $2 \mathrm{~A}$, participants reported their subjective understanding of the process by which a primary care physician (machine learning algorithm) identifies cancerous skin lesions on a scale similar to that used in study 1: "To what extent do you understand how a doctor [algorithm] examines the scans of your skin to identify cancerous skin lesions?" scored on a seven-point scale with endpoints 1 for not at all and 7 for completely understand.

In subjective understanding conditions in study $2 \mathrm{~B}$, we used a measure of subjective understanding more similar to the measure of 


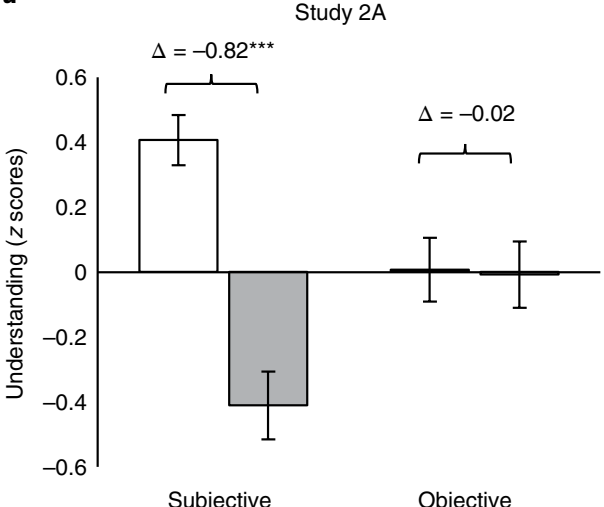

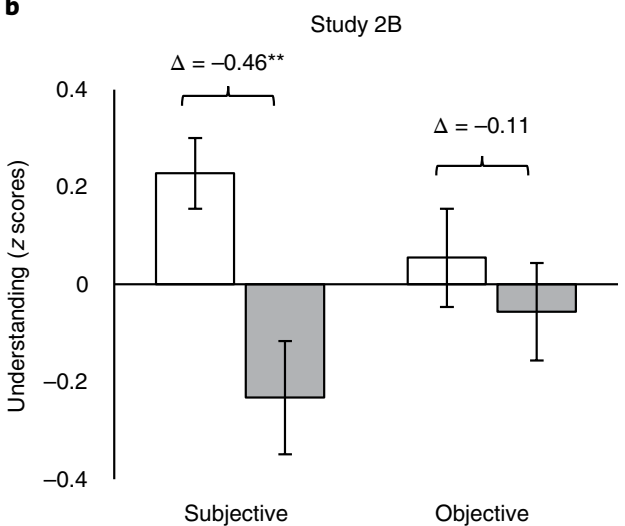

口 Human $\square$ Algorithm

$\square$ Human $\quad \square$ Algorithm

Fig. 2 | Differences in objective and subjective understanding in studies 2A,B. People report lower subjective understanding of medical decisions made by algorithmic than human providers, but exhibit similar objective understanding of decisions made by human and algorithmic providers. $\mathbf{a}, \mathbf{b}$, Study $2 \mathrm{~A}$, $N=400$ (a) and study $2 B, N=403$ (b). Error bars indicate \pm 1 s.e.m.

objective understanding, a three-item binary choice measure using more concrete descriptions of each facet of the decision process, in which participants reported whether (1) or not (0) they subjectively understood the criteria, process and output of the decision process (Supplementary Appendix 4). We scored subjective understanding in study $2 \mathrm{~B}$ by summing points for all three items; scores ranged from 0 to 3 .

We transformed raw measures of subjective and objective understanding into separate $z$ scores (Fig. 2). We then compared them by regressing understandings on provider ( 0 for algorithm, 1 for human), understanding type (0 for subjective, 1 for objective) and their interaction. Participants claimed greater subjective understanding of medical decisions made by human than algorithmic providers (2A: $\beta=-0.82, t=-6.01, P<0.001 ; 2 \mathrm{~B}: \beta=-0.46, t=-3.33$, $P=0.001$ ), whereas their objective understanding did not differ by provider (2A: $\beta=0.02, t=0.11, P=0.91 ; 2 \mathrm{~B}: \beta=0.11, t=0.79$, $P=0.43)$, creating the predicted provider $\times$ understanding interaction $(2 \mathrm{~A}: \beta=-0.80, t=-4.19, P<0.001 ; 2 \mathrm{~B}: \beta=-0.35, t=-1.78$, $P=0.08$ ). Note that, in study $2 \mathrm{~B}$, excluding the participants who failed the manipulation check increased the interaction coefficient to $\beta=-0.70, t=-2.99, P=0.003$ (Supplementary Appendix 3). Results from both studies $2 \mathrm{~A}$ and $2 \mathrm{~B}$ held when including all control variables in the regressions (Supplementary Appendix 3).

Taken together with the results of study 1, medical decisions made by both human and algorithmic providers appear to objectively be a 'black box'. However, people are more aware of their limited understanding of medical decisions made by algorithmic than human providers.

Studies 3A,B. Next, we examined whether algorithm aversion in healthcare utilization would be reduced by interventions that successfully reduced differences in the subjective understanding of medical decisions made by human and algorithmic providers. As two main decision processes are used to make mole malignancy risk assessments, and as human and algorithmic providers are each likely to favour one of these two decision processes, we again relied on a team of dermatological experts to develop two different interventions.

In study $3 \mathrm{~A}$, the intervention reduced differences in subjective understanding by explaining how all providers examine mole features (for example, asymmetry and colour) to make a malignancy risk assessment. This decision process better encapsulates how a human provider is likely to assess risk, although it is not inaccurate with respect to an algorithmic provider. In study $3 \mathrm{~B}$, the interven- tion reduced differences in subjective understanding by explaining how all providers make a malignancy risk assessment, that is, by examining the visual similarity between a target mole and other moles known to be malignant. This decision process better encapsulates how an algorithmic provider is likely to assess risk, although it is not inaccurate with respect to a human provider.

We recruited 1,599 US residents ( $3 \mathrm{~A}: N=801, M_{\mathrm{age}}=36.9$ years, $50.0 \%$ female; $3 \mathrm{~B}: N=801, M_{\text {age }}=45.3$ years, $51.1 \%$ female) from Amazon Mechanical Turk (3A) and Lucid (3B). In both studies, participants were randomly assigned to one of four conditions in a 2 (provider: human, algorithm) $\times 2$ (intervention: control, intervention) between-subject design.

We used the same skin cancer detection context as in study 1 and 2 and manipulated whether the provider was a human doctor or an algorithm (Supplementary Appendix 1). Then, participants in the control condition reported their subjective understanding and healthcare utilization intentions for that provider. Subjective understanding was measured in study $3 \mathrm{~A}$ with the item used in study $2 \mathrm{~A}$. In study $3 \mathrm{~B}$, it was measured with the item used in study 1 . Healthcare utilization intentions were measured in study $3 \mathrm{~A}$ with the item "How likely would you be to utilize a healthcare service that relies on a doctor [algorithm] to identify cancerous skin lesions?" scored on a seven-point scale with endpoints of 1 for not at all likely and 7 for very likely. In study $3 \mathrm{~B}$, they were measured with the item "How likely would you be to utilize a healthcare service that relies on a primary care physician [machine learning algorithm] to identify cancerous skin lesions?" scored on a seven-point scale with endpoints of 1 for not at all likely and 7 for very likely.

Participants in the intervention condition first rated their subjective understanding, and then read supplementary information that described how doctors [algorithms] diagnose skin cancer based on photographs of moles, in a single diagram (Fig. 3). Next, they reported their subjective understanding of the medical decision again. Finally, they reported their healthcare utilization intentions.

We first examined the effectiveness of the intervention for each kind of provider. It increased subjective understanding of the decision made by algorithmic providers (3A: $\Delta=1.42, t=12.51$, $P<0.001$; 3B: $\Delta=0.56, t=6.21, P<0.001)$ and human providers (3A: $\Delta=1.04, t=11.08, P<0.001 ; 3 \mathrm{~B}: \Delta=1.39, t=11.85, P<0.001)$. Second, we compared the extent to which the intervention reduced differences in subjective understanding across providers by comparing ratings in the control condition versus post-intervention subjective understanding ratings in the intervention condition. To this end, we regressed subjective understanding ratings on provider 
a
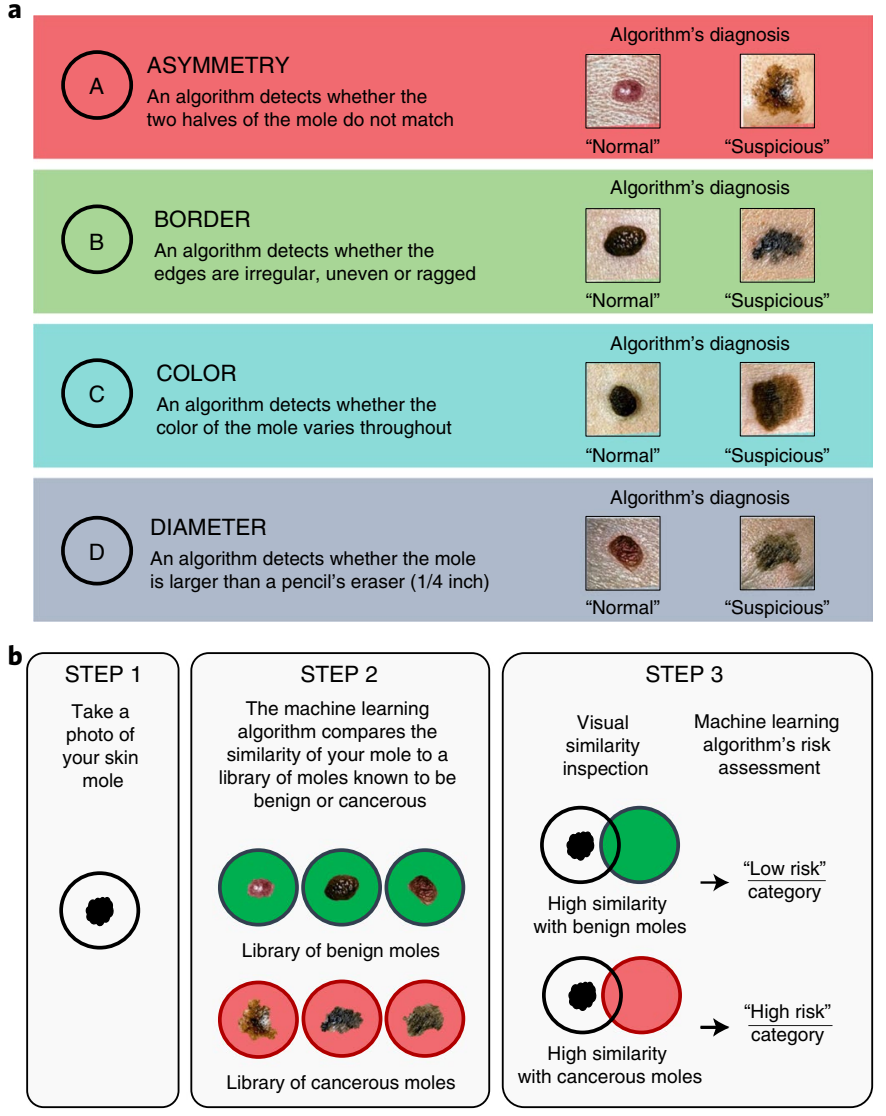

Fig. 3 | Visual interventions in studies $\mathbf{3 A}, \mathbf{B}$. a,b, Interventions in study $3 \mathrm{~A}(\mathbf{a})$ and $3 \mathrm{~B}$ (b) from the algorithm condition. In the human provider conditions, we used the same visuals and replaced "algorithm" with "doctor" (study 3A) or "machine learning algorithm" with "primary care physician" (study 3B). Mole images are in the public domain, source: National Cancer Institute (https://visualsonline.cancer.gov).

( 0 for algorithm, 1 for human), intervention ( 0 for control versus 1 for intervention) and their interaction. We found a significant provider type $\times$ intervention interaction (3A: $\beta=-0.47, t=-2.60$, $P=0.01$; 3B: $\beta=-1.02, t=-4.57, P<0.001$ ) (Fig. $4 \mathrm{a}, \mathrm{c}$ ). The difference in subjective understanding of the decision made by the human and algorithmic provider was smaller in the intervention condition (3A: $\beta=-0.70, t=-5.41, P<0.001 ; 3 \mathrm{~B}: \beta=-1.10, t=-6.97$, $P<0.001)$ than in the control condition $(3 \mathrm{~A}: \beta=-0.22, t=-1.74$, $P=0.08$; 3B: $\beta=-0.08, t=-0.52, P=0.60)$.

We next tested the impact of the intervention on algorithm aversion in healthcare utilization. We regressed utilization intentions on provider type, intervention condition and their interaction. As predicted, this revealed a significant provider type $\times$ intervention interaction (3A: $\beta=-0.49, t=-2.70, P=0.007 ; 3 \mathrm{~B}: \beta=-0.55$, $t=-2.31, P=0.02$ ) (Fig. 4b,d). Algorithm aversion in healthcare utilization (greater reluctance to utilize algorithmic than human providers) was smaller in the intervention condition (3A: $\beta=-0.58$, $t=-4.50, P<0.001$; 3B: $\beta=-0.01, t=-0.05, P=0.96)$ than in the control condition $(3 \mathrm{~A}: \beta=-1.08, t=-8.32, P<0.001$; $3 \mathrm{~B}: \beta=-0.57$, $t=-3.31, P=0.001)$. In other words, the intervention increased utilization intentions to a greater extent for algorithmic providers (3A: $\beta=0.83, t=6.46, P<0.001 ; 3 \mathrm{~B}: \beta=0.70, t=4.09, P<0.001)$ than for human providers (3A: $\beta=0.34, t=2.66, P=0.008$; $3 \mathrm{~B}$ : $\beta=0.14$, $t=0.82, P=0.41)$.

Finally, we tested our full process account by examining whether (i) subjective understanding mediated the influence of healthcare provider on utilization intentions and (ii) whether the increase in subjective understanding due to the intervention reduced algorithm aversion. We estimated a moderated mediation model of healthcare utilization with healthcare provider as predictor, intervention condition as moderator and subjective understanding as mediator with 5,000 bootstrap samples ${ }^{27}$. Consistent with our prediction, the indirect effect of healthcare provider on utilization intentions through subjective understanding was significantly stronger (3A: $\Delta=0.20,95 \%$ CI $[0.05,0.37]$; 3B: $\Delta=0.60,95 \% \mathrm{CI}$ $[0.35,0.89])$ in the control condition $(3 \mathrm{~A}: \beta=0.29,95 \%$ CI $[0.16$, $0.45]$; 3B: $\beta=0.65,95 \%$ CI $[0.45,0.86])$ where differences in subjective understanding were larger, than in the intervention condition $(3 \mathrm{~A}: \beta=0.09,95 \% \mathrm{CI}[0.02,0.17] ; 3 \mathrm{~B}: \beta=0.09,95 \% \mathrm{CI}[-0.12$, $0.21])$. These results hold when including all control variables in the regression (Supplementary Appendix 3). We acknowledge the limitations of this type of mediation analysis ${ }^{28}$ and invite future research to replicate this finding with alternative manipulations, designs and measures.

To rule out that the effects of the interventions in studies $3 \mathrm{~A}, \mathrm{~B}$ were due to differences in objective understanding between the control and intervention conditions, we ran an additional three (intervention: control, study $3 \mathrm{~A}$, study $3 \mathrm{~B}) \times 2$ (understanding: subjective, objective) between-subjects pre-registered experiment with 601 participants on Amazon Mechanical Turk. It compared both subjective and objective understanding between control conditions and two conditions that each tested one of the interventions. Results from this experiment show that, relative to a control condition, the interventions used in studies $3 \mathrm{~A}$ and $3 \mathrm{~B}$ significantly increased subjective understanding (respectively, $\beta=0.67, t=4.90, P<0.001 ; \beta=0.82, t=6.05, P<0.001)$ but did not influence objective understanding (respectively, $P=0.78$ and $P=0.89$; all interactions, $t s>3.60, p s<0.001)$. For detailed study descriptions and results, see Supplementary Appendix 5. We acknowledge that the results of this ancillary study may have been influenced by the sensitivity of our measure of objective understanding. Measures with a wider range of easy and hard questions might identify more subtle changes in objective understanding resulting from these or other interventions, and identify complementary effects of changes in objective understanding on utilization of medical AI.

Study 4. In study 4, using Google Ads, we tested whether subjective knowledge-based interventions would effectively reduce algorithm aversion in healthcare utilization in an online field setting. Following Winterich et al. ${ }^{29}$, we published sponsored search advertisements for an algorithm-based skin cancer detection application (Fig. 5). Whenever a user typed a prespecified search keyword (for example, "skin cancer picture") they might see our sponsored search advertisement near or above the organic results on Google (see Supplementary Appendix 6 for details).

We ran our ad campaign for five days between 15 and 19 November 2020. We specified a daily budget of 60 euros; on each day our advertisements stopped when the daily budget was reached. The advertisements generated 14,013 impressions (that is, how many times the ad was viewed across the following demographics: age: $18-24$ years: $6.9 \%, 25-35$ years: $5.7 \%, 35-44$ years: $7.3 \%, 45-54$ years: $15.1 \%$, $55-64$ years: $35.6 \%$, $65+$ years: $29.4 \%$; gender: $78.8 \%$ female) and generated 698 clicks.

All participants were served one of two ads for a skin cancer detection application through Google Ads (Fig. 5). We manipulated whether the sponsored ad did or did not explain the process by which the algorithm triaged moles and tested the impact of that intervention on ad click-through rates. Subjective understanding was manipulated through these descriptions of the application, as pretested in a study reported in Supplementary Appendix 7. In the intervention condition, the advertisement read "Our algorithm 


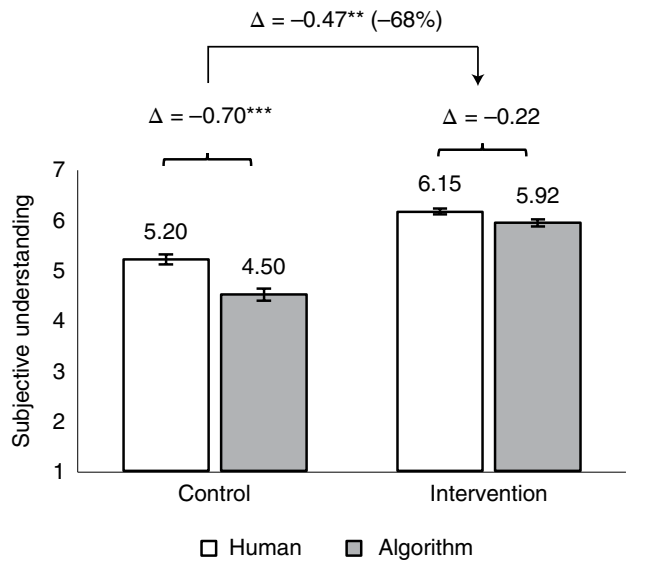

C

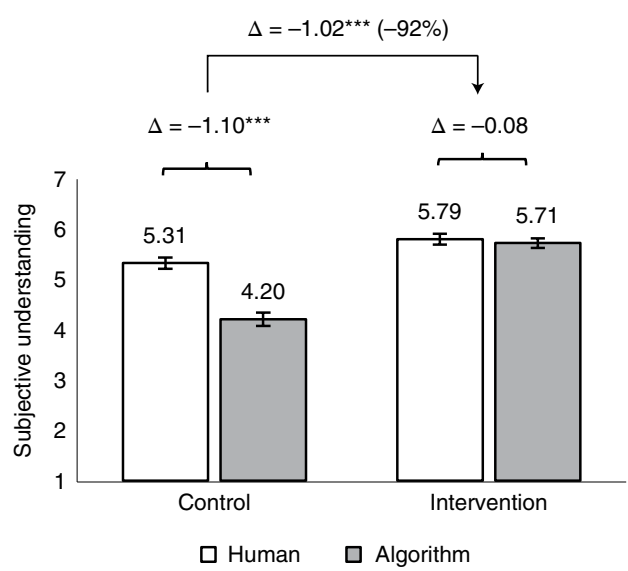

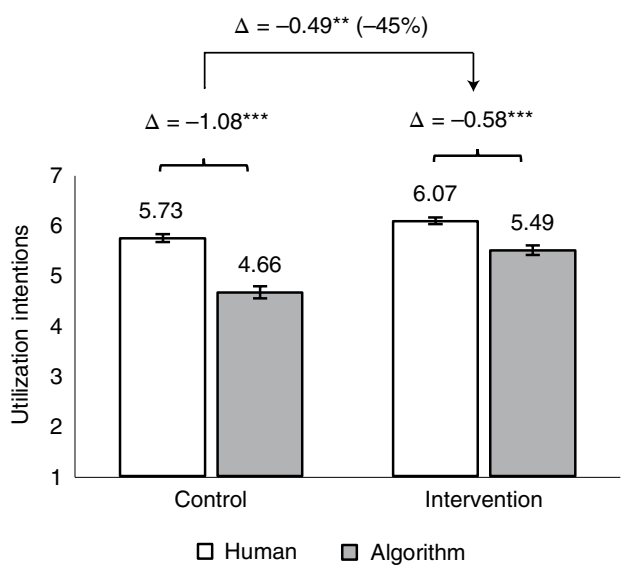

d

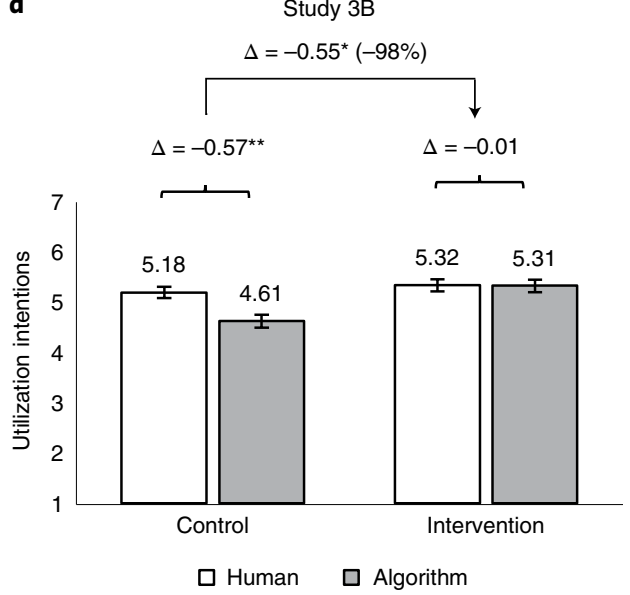

Fig. 4 | Effectiveness of interventions in studies 3A,B. a-d, Explanations reduce the difference in subjective understanding between algorithmic and human decision-making in study $3 \mathrm{~A}$ (a) and 3B (c), with scale endpoints marked as 1 for not at all and 7 for completely understand, which increases utilization intentions for the algorithmic provider in study $3 \mathrm{~A}$ (b) and 3B (d), with scale endpoints marked with 1 for not at all likely and 7 for very likely. $N=801$ and $N=798$ in studies $3 A$ and $3 B$, respectively. Error bars indicate \pm 1 s.e.m.

checks how similar skin moles are in shape/size/color to cancerous moles." In the control condition, the advertisement read "Our algorithm checks if skin moles are cancerous moles." Clicking on the advertisement took participants to the Google App Playstore page of SkinVision, an algorithmic skin cancer detection app (Supplementary Appendices 6 and 8).

The frequency of appearance of the different ads was not random, because the Google Ads platform serves ads according to the goal of the campaign (maximizing clicks, in our case). Hence, we cannot use the actual number of clicks as a dependent variable. Instead, we followed prior research ${ }^{29}$ and used the average percentage of clicks per impression or click-through rate. A logistic regression revealed that the click-through rate was higher in the intervention advertisement $(6.36 \%)$ compared with the control advertisement $(3.29 \%$, $\beta=0.69, z=8.17, P<0.001, d=0.38)$.

The results illustrate the efficacy of advertising interventions enhancing subjective knowledge in field settings. Because the assignment of users between ads was not random, of course, it may have generated unintended variance in the set of users exposed to each $\mathrm{ad}^{30}$. Given the replications in studies $3 \mathrm{~A}, \mathrm{~B}$, which featured random assignment, we believe it is unlikely that the results from study 4 can be solely attributed to ad optimization. We invite future research to further replicate this result by using different methodologies and sampling procedures.

\section{Discussion}

Utilization of algorithmic-based healthcare services is becoming critical with the rise of telehealth services ${ }^{31}$, the current surge in healthcare demand $\mathrm{d}^{2-4}$ and long-term goals of providing affordable and high-quality healthcare ${ }^{32}$ in developed and developing nations ${ }^{9}$. Our results yield practical insights for reducing reluctance to utilize medical AI. Because the technologies used in algorithmic-based medical applications are complex, providers tend to present AI provider decisions as a 'black box'. Our results underscore the importance of recent policy recommendations to open this black box to patients and users ${ }^{16,33}$. A simple one-page visual or sentence that explains the criteria or process used to make medical decisions increased acceptance of an algorithm-based skin cancer diagnostic tool, which could be easily adapted to other domains and procedures.

Given the complexity of the process by which medical AI makes decisions, firms now tend to emphasize the outcomes that algorithms produce in their marketing to consumers, which feature benefits such as accuracy, convenience and rapidity (performance), while providing few details about how algorithms work (process). Indeed, in an ancillary study examining the marketing of skin cancer smartphone applications (Supplementary Appendix 8), we find that performance-related keywords were used to describe $57-64 \%$ of the applications, whereas process-related keywords were used to 
Skin Cancer Detection app I Checks mole

Ad · play.google.com

Our algorithm checks if skin moles are cancerous moles. Take pictures of your skin with this smartphone app and get a skin cancer risk assessment

\section{b}

Skin Cancer Detection App I Checks mole shape/size/color Ad · play.google.com

Our algorithm checks how similar skin moles are in shape/size/color to cancerous moles. Take pictures of your skin with this smartphone app and get a skin cancer risk assessment

Fig. 5 | Google Ads displayed in study 4. a,b, Control (a) and intervention (b) sponsored search advertisements published on the Google Ads platform ( $N=14,013$ impressions).

describe $21 \%$ of the applications. Improving subjective understanding of how medical AI works may then not only provide beneficent insights for increasing consumer adoption but also for firms seeking to improve their positioning. Indeed, we find increased advertising efficacy for SkinVision, a skin cancer detection app, when advertising included language explaining how it works.

More broadly, our findings make theoretical contributions to the literatures on algorithm aversion and human understanding of causal systems. The literature on algorithm aversion finds that people are generally averse to using algorithms for tasks that are usually done by humans ${ }^{34,35}$, on the grounds that algorithms are perceived as unable to learn and improve from their mistakes ${ }^{36}$, unsuitable for subjective or experiential tasks ${ }^{37,38}$, unable to adapt to unique or mutable circumstances ${ }^{10,39}$ and unable to carry responsibility for negative outcomes ${ }^{11}$. Our results illustrate the importance of understanding the causal process relating their inputs (for example, medical data) to their outputs (for example, diagnoses). We also identify interventions to reduce algorithm aversion in a domain where algorithms are already in widespread use ${ }^{1}$. We invite future research on medical AI adoption with alternative and complementary interventions of health literacy ${ }^{40}$ or digital literacy ${ }^{41}$. Finally, people do exhibit an illusion of explanatory depth of causal systems ${ }^{21-23,42}$ in many domains, claiming to better understand a variety of ideas, technologies and biological systems than they objectively do. Our results suggest an egocentric bias in this illusion of understanding, that the illusion may loom largest in assessments of people-the causal systems most similar to ourselves.

\section{Methods}

The present research involved no more than minimal risks, and all study participants were 18 years of age or older. Studies 1-3B were approved for use with human participants by the Institutional Review Board on the Charles River Campus at Boston University (protocol 3632E); informed consent was obtained for all participants. Study 4 used data collected from the Google Ads platform, which is aggregated and fully anonymous at the individual level. It is impossible to identify, interact with and obtain consent from individual participants. This study was approved by the Erasmus University Erasmus Research Institute of Management Internal Review Board.

All manipulations and measures are reported. Pre-registrations, raw data and Stata syntax files are available on the Open Science Framework at https://osf.io/ taqp8/.

In studies 1-3B, we recruited participants from the online sample recruiting platforms Lucid and Amazon Mechanical Turk. Lucid provides a nationally representative sample with respect to age, gender, ethnicity and geography ${ }^{43}$. Amazon Mechanical Turk is an online sample recruiting platform that, although not nationally representative, yields high data quality through the use of attention checks $^{44}$ and worker reputation scores ${ }^{45}$. Following prior research, we selected participants with an approval rating above 95\% on Amazon Mechanical Turk ${ }^{45}$. Studies 1-3B were conducted on the Qualtrics survey platform. Condition assignments were random in all our studies, with randomization administered by Qualtrics software.

Following a general rule of thumb used in recent research ${ }^{46}$, we sought to obtain a minimum of 100 participants per cell. As a result, in studies $2 \mathrm{~A}, \mathrm{~B}$, we decided to target a sample of 100 per cell. In study 1 we decided to target a larger sample of 150 per cell to have sufficient power to detect an interaction in a $2 \times$ 2 mixed design (one factor was manipulated between subject while the other was repeated within subjects). In studies $3 \mathrm{~A}, \mathrm{~B}$, we decided to target a sample of 200 per cell to have sufficient power to detect moderated mediation. With a small effect size of Cohen's $f=0.20$, a significance level of 0.05 and $n=100$ in two between-subject conditions, the power to detect a significant effect was $80.36 \%$. With sample sizes of $n=150$ and $n=200$, the power was $93.23 \%$ and $97.88 \%$, respectively.

In studies 1, 2A and 3A,B, as pre-registered, we programmed our surveys to automatically exclude participants who failed an attention check at the very beginning of the survey and prior to any manipulation. We have no data for these participants, and because these responses are recorded as incomplete, they did not affect our target sample size. Participants answered the following attention check question: "There is an artist named Frank that paints miniature figures. He usually buys these figures from a company, but the company has gone out of business. After this, Frank decides to hand-carve his own figures. However, Frank's friend tells him that these new figures are significantly worse in quality. Did Frank's friend think that Frank's hand-carved figures were better than the company's?" with answers of yes, no, maybe, cheese plates and movies (correct answer is "no"). We screened out $N=211$ participants in study $1, N=255$ participants in study $2 \mathrm{~A}$, $N=260$ participants in study $3 \mathrm{~A}$ and $N=378$ participants in study $3 \mathrm{~B}$.

In studies $1,2 \mathrm{~A}$ and $3 \mathrm{~A}, \mathrm{~B}$, the stimuli included either an image of a human provider or an algorithm, the first result in Google Images with search terms "Doctor" or "Artificial Intelligence" that did not include words or a face (see https://osf.io/taqp8/). Omitting these introductory images in study $2 \mathrm{~B}$ yielded the same pattern of results.

Based on prior research on resistance to $\mathrm{AI}$ in the medical domain ${ }^{10}$, participants also reported three control variables related to skin cancer in studies 1-3B: their perceived susceptibility to skin cancer ("Relative to an average person of your same age and gender, to what extent do you consider yourself to be at risk of skin cancer (melanoma)?" with 1 for much lower and 5 for much higher), their self-examination frequency ("Skin self-examination is the careful and deliberate checking for changes in spots or moles on all areas of your skin, including those areas rarely exposed to the sun. How often do you practice skin self-examination?" with 1 for never and 5 for weekly) and their perceived self-efficacy ("In general, to what extent do you feel that you are confident in your ability to conduct skin self-examination?" with 1 for not at all confident and 5 for extremely confident).

In studies 1-3B, we also collected demographic variables at the end of the experiment (gender, age and employment by/as a healthcare provider) and a manipulation check question ("In the scenario you read, the data [...] was analyzed by?" with answers of a doctor or an algorithm). Additional details about the manipulation check, and analyses including the control variables, are described in Supplementary Appendix 3. Effect sizes associated with main hypotheses are detailed in Supplementary Appendix 9.

Reporting summary. Further information on research design is available in the Nature Research Reporting Summary linked to this article.

\section{Data availability}

Data from all of the studies reported in this paper are publicly available at https:// osf.io/taqp8/.

\section{Code availability}

Analyses were conducted with STATA 16.1, and code from all studies is publicly available at https://osf.io/taqp8/.

Received: 21 December 2020; Accepted: 27 May 2021; Published online: 28 June 2021

\section{References}

1. Topol, E. J. High-performance medicine: the convergence of human and artificial intelligence. Nat. Med. 25, 44-56 (2019).

2. Wosik, J. et al. Telehealth transformation: COVID-19 and the rise of virtual care. J. Am. Med. Inform. Assoc. 27, 957-962 (2020).

3. Hollander, J. E. \& Carr, B. G. Virtually perfect? Telemedicine for COVID-19. N. Engl. J. Med. 382, 1679-1681 (2020).

4. Keesara, S., Jonas, A. \& Schulman, K. Covid-19 and health care's digital revolution. N. Engl. J. Med. 382, e82 (2020).

5. Esteva, A. et al. Dermatologist-level classification of skin cancer with deep neural networks. Nature 542, 115-118 (2017).

6. Laranjo, L. et al. Conversational agents in healthcare: a systematic review. J. Am. Med. Inform. Assoc. 25, 1248-1258 (2018). 
7. Goto, T., Camargo, C. A. Jr, Faridi, M. K., Freishtat, R. J. \& Hasegawa, K. Machine learning-based prediction of clinical outcomes for children during emergency department triage. JAMA Netw. Open 2, e186937-e186937 (2019).

8. Hao, K. Doctors are using AI to triage covid-19 patients. The tools may be here to stay. MIT Technology Review (23 April 2020).

9. Guo, J. \& Li, B. The application of medical artificial intelligence technology in rural areas of developing countries. Health Equity 2, 174-181 (2018).

10. Longoni, C., Bonezzi, A. \& Morewedge, C. K. Resistance to medical artificial intelligence. J. Cons. Res. 46, 629-650 (2019).

11. Promberger, M. \& Baron, J. Do patients trust computers? J. Behav. Decis. Mak. 19, 455-468 (2006).

12. Eastwood, J., Snook, B. \& Luther, K. What people want from their professionals: attitudes toward decision-making strategies. J. Behav. Decis. Mak. 25, 458-468 (2012).

13. Price, W. N. Big data and black-box medical algorithms. Sci. Transl. Med. 10, eaao5333 (2018)

14. Burrell, J. How the machine 'thinks': understanding opacity in machine learning algorithms. Big Data Soc. 3, 2053951715622512 (2016).

15. Castelvecchi, D. Can we open the black box of AI? Nature 538, 20-23 (2016)

16. Kroll, J. A. et al. Accountable algorithms. Univ. Pa. Law Rev. 165, 633 (2016).

17. Nisbett, R. E. \& Wilson, T. D. Telling more than we can know: verbal reports on mental processes. Psychol. Rev. 84, 231-259 (1977).

18. Kahneman, D. Maps of bounded rationality: psychology for behavioral economics. Am. Econ. Rev. 93, 1449-1475 (2003).

19. Morewedge, C. K. \& Kahneman, D. Associative processes in intuitive judgment. Trends Cogn. Sci. 14, 435-440 (2010).

20. Pronin, E. \& Kugler, M. B. Valuing thoughts, ignoring behavior: the introspection illusion as a source of the bias blind spot. J. Exp. Soc. Psychol. 43, 565-578 (2007).

21. Fernbach, P. M., Sloman, S. A., Louis, R. S. \& Shube, J. N. Explanation fiends and foes: how mechanistic detail determines understanding and preference. $J$. Cons. Res. 39, 1115-1131 (2013).

22. Fernbach, P. M., Rogers, T., Fox, C. R. \& Sloman, S. A. Political extremism is supported by an illusion of understanding. Psychol. Sci. 24, 939-946 (2013).

23. Rozenblit, L. \& Keil, F. The misunderstood limits of folk science: an illusion of explanatory depth. Cogn. Sci. 26, 521-562 (2002).

24. Stolz, W. ABCD rule of dermatoscopy: a new practical method for early recognition of malignant melanoma. Eur. J. Dermatol. 4, 521-527 (1994).

25. Rogers, T. et al. A clinical aid for detecting skin cancer: the triage amalgamated dermoscopic algorithm (TADA). J. Am. Board Fam. Med. 29, 694-701 (2016).

26. Robinson, J. K. et al. A randomized trial on the efficacy of mastery learning for primary care provider melanoma opportunistic screening skills and practice. J. Gen. Intern. Med. 33, 855-862 (2018).

27. Hayes, A. F. Introduction to Mediation, Moderation, and Conditional Process Analysis: A Regression-Based Approach (Guilford Press, 2013).

28. Bullock, J. G., Green, D. P. \& Ha, S. E. Yes, but what's the mechanism? (don't expect an easy answer). J. Pers. Soc. Psychol. 98, 550 (2010).

29. Winterich, K. P., Nenkov, G. Y. \& Gonzales, G. E. Knowing what it makes: how product transformation salience increases recycling. J. Mark. 83, 21-37 (2019).

30. Eckles, D., Gordon, B. R. \& Johnson, G. A. Field studies of psychologically targeted ads face threats to internal validity. Proc. Natl Acad. Sci. USA 115, E5254-E5255 (2018)

31. Tuckson, R. V., Edmunds, M. \& Hodgkins, M. L. Telehealth. N. Engl. J. Med. 377, 1585-1592 (2017)

32. Reinhard, S. C., Kassner, E. \& Houser, A. How the Affordable Care Act can help move states toward a high-performing system of long-term services and supports. Health Aff. 30, 447-453 (2011).

33. Watson, D. S. et al. Clinical applications of machine learning algorithms: beyond the black box. BMJ 364, 1886 (2019).
34. Dawes, R., Faust, D. \& Meehl, P. Clinical versus actuarial judgment. Science 243, 1668-1674 (1989).

35. Yeomans, M., Shah, A., Mullainathan, S. \& Kleinberg, J. Making sense of recommendations. J. Behav. Decis. Mak. 32, 403-414 (2019).

36. Dietvorst, B. J., Simmons, J. P. \& Massey, C. Algorithm aversion: people erroneously avoid algorithms after seeing them err. J. Exp. Psychol. Gen. 144, 114 (2015).

37. Castelo, N., Bos, M. W. \& Lehmann, D. R. Task-dependent algorithm aversion. J. Mark. Res. 56, 809-825 (2019).

38. Longoni, C. \& Cian, L. Artificial intelligence in utilitarian vs. hedonic contexts: the "word-of-machine" effect. J. Market. https://doi. org/10.1177/0022242920957347 (2020).

39. Dietvorst, B. J. \& Bharti, S. People reject algorithms in uncertain decision domains because they have diminishing sensitivity to forecasting error. Psychol. Sci. 31, 1302-1314 (2020).

40. Ayre, J., Bonner, C., Cvejic, E. \& McCaffery, K. Randomized trial of planning tools to reduce unhealthy snacking: implications for health literacy. PLoS ONE 14, e0209863 (2019).

41. Neter, E. \& Brainin, E. eHealth literacy: extending the digital divide to the realm of health information. J. Med. Internet Res. 14, e19 (2012).

42. Alter, A. L., Oppenheimer, D. M. \& Zemla, J. C. Missing the trees for the forest: a construal level account of the illusion of explanatory depth. J. Pers. Soc. Psychol. 99, 436 (2010).

43. Pennycook, G. \& Rand, D. G. Fighting misinformation on social media using crowdsourced judgments of news source quality. Proc. Natl Acad. Sci. USA 116, 2521-2526 (2019).

44. Paolacci, G., Chandler, J. \& Ipeirotis, P. G. Running experiments on Amazon Mechanical Turk. Judgm. Decis. Mak. 5, 411-419 (2010).

45. Peer, E., Vosgerau, J. \& Acquisti, A. Reputation as a sufficient condition for data quality on Amazon Mechanical Turk. Behav. Res. Methods 46, 1023-1031 (2014).

46. Hofstetter, R., Rüppell, R. \& John, L. K. Temporary sharing prompts unrestrained disclosures that leave lasting negative impressions. Proc. Natl Acad. Sci. USA 114, 11902-11907 (2017).

\section{Acknowledgements}

Before joining Rotterdam School of Management, R.C. received funding from the Susilo Institute for Ethics in the Global Economy, Questrom School of Business, Boston University. R.C. thanks the Erasmus Research Institute in Management for providing funding for data collection. The authors thank T. Sangers, M. Wakkee, A. Udrea and SkinVision for their feedback on human and algorithmic decision processes.

\section{Author contributions}

R.C., C.L. and C.K.M designed research; R.C. and C.L. performed research; R.C. analysed data; R.C., C.L. and C.K.M wrote the paper.

\section{Competing interests}

The authors declare no competing interests.

\section{Additional information}

Supplementary information The online version contains supplementary material available at https://doi.org/10.1038/s41562-021-01146-0.

Correspondence and requests for materials should be addressed to R.C.

Peer review information Nature Human Behaviour thanks Fiona Hamilton and the other, anonymous, reviewer(s) for their contribution to the peer review of this work.

Reprints and permissions information is available at www.nature.com/reprints.

Publisher's note Springer Nature remains neutral with regard to jurisdictional claims in published maps and institutional affiliations.

(c) The Author(s), under exclusive licence to Springer Nature Limited 2021 


\section{Reporting Summary}

Nature Research wishes to improve the reproducibility of the work that we publish. This form provides structure for consistency and transparency in reporting. For further information on Nature Research policies, see our Editorial Policies and the Editorial Policy Checklist.

\section{Statistics}

For all statistical analyses, confirm that the following items are present in the figure legend, table legend, main text, or Methods section.

n/a Confirmed

$\bigotimes$ The exact sample size $(n)$ for each experimental group/condition, given as a discrete number and unit of measurement

$\square$ A statement on whether measurements were taken from distinct samples or whether the same sample was measured repeatedly

$\square$ The statistical test(s) used AND whether they are one- or two-sided

$\square$ Only common tests should be described solely by name; describe more complex techniques in the Methods section.

$\square$ A description of all covariates tested

$\square$ \A description of any assumptions or corrections, such as tests of normality and adjustment for multiple comparisons

$\square$ A full description of the statistical parameters including central tendency (e.g. means) or other basic estimates (e.g. regression coefficient)

$\triangle$ AND variation (e.g. standard deviation) or associated estimates of uncertainty (e.g. confidence intervals)

$\varnothing$ For null hypothesis testing, the test statistic (e.g. $F, t, r$ ) with confidence intervals, effect sizes, degrees of freedom and $P$ value noted

Give P values as exact values whenever suitable.

Х $\square$ For Bayesian analysis, information on the choice of priors and Markov chain Monte Carlo settings

$\square$ \ For hierarchical and complex designs, identification of the appropriate level for tests and full reporting of outcomes

$\square \bigotimes$ Estimates of effect sizes (e.g. Cohen's $d$, Pearson's $r$ ), indicating how they were calculated

Our web collection on statistics for biologists contains articles on many of the points above.

\section{Software and code}

Policy information about availability of computer code

Data collection Qualtrics

Data analysis Stata

For manuscripts utilizing custom algorithms or software that are central to the research but not yet described in published literature, software must be made available to editors and reviewers. We strongly encourage code deposition in a community repository (e.g. GitHub). See the Nature Research guidelines for submitting code \& software for further information.

\section{Data}

Policy information about availability of data

All manuscripts must include a data availability statement. This statement should provide the following information, where applicable:

- Accession codes, unique identifiers, or web links for publicly available datasets

- A list of figures that have associated raw data

- A description of any restrictions on data availability

Pre-registrations, raw data and syntax files are available on the Open Science Framework at https://osf.io/taqp8/ 


\section{Field-specific reporting}

Please select the one below that is the best fit for your research. If you are not sure, read the appropriate sections before making your selection.
Life sciences
Behavioural \& social sciences
Ecological, evolutionary \& environmental sciences

For a reference copy of the document with all sections, see nature.com/documents/nr-reporting-summary-flat.pdf

\section{Behavioural \& social sciences study design}

All studies must disclose on these points even when the disclosure is negative.

Study description

Research sample

Sampling strategy

Data collection

Timing

Data exclusions

Non-participation

Randomization
Experiments (studies 1, 2A, 2B, 3A, 3B) and online advertising study (study 4)

Study 1. We recruited a nationally representative of 297 US residents (Mage $=45.37 ; 53 \%$ female) from Lucid. Study 2A. We recruited a nationally representative of 400 US residents (Mage $=45.36,52 \%$ female) from Lucid. Study 2B. We recruited a nationally representative of 403 US residents (Mage $=45.16,52 \%$ female) from Lucid.

Studies 3A-B. We recruited 1599 US residents (3A: $N=801$, Mage $=36.9,50.0 \%$ female and 3B: $N=801$, Mage $=45.3,51.1 \%$ female) from Amazon Mechanical Turk (3A) and Lucid (3B).

Study 4. Google ads which generated 14,013 impressions in total (age: 18-24: 6.9\%, 25-35: 5.7\%, 35-44: 7.3\%, 45-54: 15.1\%, 55-64: $35.6 \%, 65+$ : 29.4\%; gender: $78.8 \%$ female)

Minimum of 100 per experimental condition.

Studies 1, 2A, 2B, 3A, 3B: Qualtrics with online panels

Studiy 4: Google Ads

Study 1. July, 2020

Study 2A, Oct 2020

Study 2B, March 2021

Study 3A, June 2020

Study 3B, Oct 2020

Study 4. Nov 2020

No exclusions in data analyses

No participant declined participation

Studies 1, 2A, 2B, 3A, 3B: Qualtrics randomizer

Study 4: Allocation from Google platform

\section{Reporting for specific materials, systems and methods}

We require information from authors about some types of materials, experimental systems and methods used in many studies. Here, indicate whether each material, system or method listed is relevant to your study. If you are not sure if a list item applies to your research, read the appropriate section before selecting a response.

Materials \& experimental systems

\begin{tabular}{|c|c|}
\hline$n / a$ & Involved in the study \\
\hline Х & Antibodies \\
\hline$凶$ & Eukaryotic cell lines \\
\hline$\bigotimes$ & Palaeontology and archaeology \\
\hline$\bigotimes$ & $\square$ Animals and other organisms \\
\hline & \ Human research participants \\
\hline$凶$ & $\square$ Clinical data \\
\hline$凶$ & $\square$ Dual use research of concern \\
\hline
\end{tabular}

\section{Human research participants}

Policy information about studies involving human research participants

Population characteristics

See above

Recruitment

Lucid, Amazon Mechanical Turk, Google Ads

\begin{tabular}{l|l}
\multicolumn{2}{l}{ Methods } \\
\hline n/a & Involved in the study \\
$\searrow$ & $\square$ ChIP-seq \\
$\searrow$ & $\square$ Flow cytometry \\
$\square$ & $\square$ MRI-based neuroimaging
\end{tabular}


Ethics oversight

Boston University, Erasmus University

Note that full information on the approval of the study protocol must also be provided in the manuscript.

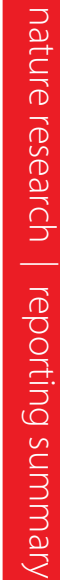

$\frac{8}{0}$

3 